www.asianjournalofmycology.org Article

Doi 10.5943/ajom/2/1/21

\title{
Minutisphaera aquaticum sp. nov. increases the known diversity of Minutisphaeraceae
}

\author{
Bao DF ${ }^{1,2,3}$, Hyde $\mathrm{KD}^{2}$, Luo ZL ${ }^{1}$, Su HY ${ }^{1}$, Nalumpang $\mathrm{S}^{3^{*}}$ \\ ${ }^{1}$ College of Agriculture and Biological Sciences, Dali University, Dali 671003, Yunnan, P.R. China \\ ${ }^{2}$ Center of Excellence in Fungal Research, Mae Fah Luang University, Chiang Rai 57100, Thailand \\ ${ }^{3}$ Department of Entomology and Plant Pathology, Faculty of Agriculture, Chiang Mai University, Chiang Mai 50200, \\ Thailand
}

Bao DF, Hyde KD, Luo ZL, Su HY, Nalumpang S 2019 - Minutisphaera aquaticum sp. nov. increases the known diversity of Minutisphaeraceae. Asian Journal of Mycology 2(1), 306-314, Doi 10.5943/ajom/2/1/21

\begin{abstract}
New species of freshwater fungi are constantly being introduced following our studies in Asia. In the present paper, Minutisphaera aquaticum sp. nov., is introduced from submerged wood collected in the Mekong River in eastern Thailand, and increases the known diversity of Minutisphaeraceae. Minutisphaera aquaticum is characterized by superficial, small globose, dark brown to black ascomata, bitunicate, fissitunicate, obovoid to broadly cylindrical asci and fusiform, hyaline ascospores with a supra-median primary septum and upper cells that are wider than the lower cells. The multigene phylogenetic analysis places the new taxon in a well-supported clade with the species in Minutisphaeraceae (Minutisphaerales). The new species is compared with other Minutisphaera species, description and illustration are provided.
\end{abstract}

Key words - 1 new species - Minutisphaerales - molecular - phylogeny - taxonomy

\section{Introduction}

Minutisphaerales, an order of freshwater ascomycetes within the class Dothideomycetes, was recently established by Raja et al. (2015) to accommodate the monotypic genus Minutisphaera. Minutisphaerales currently comprises one family, Minutisphaeraceae, with four species and were reported from freshwater habitats in Japan and the USA.

The genus Minutisphaera was introduced by Ferrer et al. (2010) with a single species $M$. fimbriatispora in the class Dothideomycetes. Members of this genus are characterized by small, globose to subglobose or apothecioid, erumpent to superficial, brown ascomata, fissitunicate, eightspored, ovoid to obclavate asci, and 1-2-septate, clavate to broadly fusiform, hyaline to pale brown ascospores, with or without a gelatinous sheath and filamentous appendages. Three additional species, viz. M. japonica, M. fimbriatispora and M. aspera, were later added (Raja et al. 2013, 2015).

In a recent study, lignicolous freshwater fungi were studied along the north-south gradient in the Asian/Australian regions (Hyde et al. 2016). The new taxon, Minutisphaera aquaticum is described, illustrated and compared with similar taxa in this article. Phylogenetic analyses of combined LSU, SSU, and ITS sequence data provide evidence for the new species and confirms its placement in Minutisphaera. 


\section{Collection and examination of specimens}

Specimens of submerged decaying wood were collected from Mekong River in Nakhon Phanom province, Thailand. Specimens were brought to the laboratory in plastic bags and incubated in plastic boxes lined with moistened tissue paper at room temperature for one week. Sample examination and morphological studies followed the protocols outlined previously (Luo et al. 2018). Single spore isolations were made on to potato dextrose agar (PDA) and later transferred on to fresh PDA following the method of Chomnunti et al. (2014). Specimens (dry wood with fungal material) are deposited in the herbarium of Mae Fah Luang University (MFLU), Chiang Rai, Thailand. Axenic cultures are deposited in Mae Fah Luang University Culture Collection (MFLUCC). Faces of Fungi and Index Fungorum numbers are registered as outlined in Jayasiri et al. (2015) and Index Fungorum (2018).

\section{DNA extraction, PCR amplification and sequencing}

Isolates were grown on PDA medium at $25^{\circ} \mathrm{C}$ for one month. Fungal mycelium was scraped off and transferred to a $1.5 \mathrm{ml}$ microcentrifuge tubes using a sterilized lancet for genomic DNA extraction. Ezup Column Fungi Genomic DNA Purification Kit (Sangon Biotech, China) was used to extract DNA following the manufacturer's instructions. The internal transcribed spacer (ITS), large-subunit rRNA (LSU) and small-subunit ribosomal RNA (SSU) gene regions were amplified using the primer pairs ITS5/ITS4 (Vilgalys \& Hester 1990), LROR/LR7 and NS1/ NS4 (White et

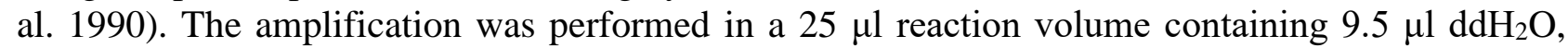
$12.5 \mu \mathrm{l} 2 \times$ Taq PCR Master Mix with blue dye (Sangon Biotech, China), $1 \mu$ of DNA template and $1 \mu \mathrm{l}$ of each primer $(10 \mu \mathrm{M})$. The amplification condition for ITS, LSU and SSU consisted of initial denaturation at $94{ }^{\circ} \mathrm{C}$ for $3 \mathrm{~min}$; followed by 40 cycles of $45 \mathrm{~s}$ at $94{ }^{\circ} \mathrm{C}, 50 \mathrm{~s}$ at $56{ }^{\circ} \mathrm{C}$ and 1 min at $72{ }^{\circ} \mathrm{C}$ and a final extension period of $10 \mathrm{~min}$ at $72{ }^{\circ} \mathrm{C}$. Purification and sequencing of PCR products were carried out using the above-mentioned PCR primers at Sangon Biotech (Shanghai) Co. Ltd. in China.

\section{Phylogenetic analyses}

The taxa included in the phylogenetic analyses were selected and obtained from previous studies (Raja et al. 2015, Liu et al. 2017) and GenBank (Table 1). Three gene regions (ITS, LSU and SSU) were used for the combined sequence data analyses. SEQMAN v. 7.0.0 (DNASTAR, Madison, WI) was used to assemble consensus sequences. The sequences were aligned using the online multiple alignment program MAFFT v.7 (http://mafft.cbrc.jp/alignment/server/) (Katoh \& Standley 2013). The alignments were checked visually and improved using BioEdit.

Maximum likelihood (ML) analysis was performed using RAxML-HPC v.8 (Stamatakis 2006, Stamatakis et al. 2008) on the XSEDE Teragrid of the CIPRES Science Gateway (https://www.phylo.org) (Miller et al. 2010) with rapid bootstrap analysis, followed by 1000 bootstrap replicates. The final tree was selected amongst suboptimal trees from each run by comparing likelihood scores under the GTRGAMMA substitution model.

Maximum parsimony (MP) analyses were performed with PAUP v. 4.0b10 (Swofford 2003) using the heuristic search option with 1000 random taxa addition and tree bisection and reconnection (TBR) as the branch swapping algorithm. All characters were unordered and of equal weight and gaps were treated as missing data. Maxtrees were unlimited, branches of zero length were collapsed and all multiple, equally parsimonious trees were saved. Clade stability was assessed using a bootstrap (BT) analysis with 1000 replicates, each with 10 replicates of random stepwise addition of taxa (Hillis \& Bull 1993).

The program MRMODELTEST2 v. 2.3 (Nylander 2004) was used to infer the appropriate substitution model that would best fit the model of DNA evolution for the combined datasets for Bayesian inference analysis with $\mathrm{GTR}+\mathrm{G}+\mathrm{I}$ substitution model selected. Posterior probabilities (PP) (Rannala \& Yang 1996, Zhaxybayeva \& Gogarten 2002) were determined by Markov Chain 
Monte Carlo sampling (MCMC) in MRBAYES v. 3.0b4 (Huelsenbeck \& Ronquist 2001). Six simultaneous Markov chains were run for 1 million generations, with trees sampled every 100 generations (resulting in 10000 trees). The first 2000 trees, representing the burn-in phase of the analyses were discarded and the remaining 8000 trees were used for calculating posterior probabilities (PP) in the majority rule consensus tree (Larget \& Simon 1999).

Phylogenetic trees were represented by FigTree v. 1.4.0 (Rambaut 2012) and edited in Microsoft Office PowerPoint 2016 (Microsoft Inc., United States). Newly generated sequences in this study were deposited in GenBank (Table 1) and the final matrices used for the phylogenetic analyses were submitted to TreeBASE (www.treebase.org; accession number: 25572).

Table 1 GenBank and culture collection accession numbers of species included in the phylogenetic study. The newly generated sequences are shown in bold.

\begin{tabular}{|c|c|c|c|c|}
\hline \multirow{2}{*}{ Taxa } & \multirow{2}{*}{ Strain no. } & \multicolumn{3}{|c|}{ GenBank accession numbers } \\
\hline & & ITS & LSU & SSU \\
\hline Aliquandostipite khaoyaiensis & CBS 118232 & JN942357 & GU301796 & AF201453 \\
\hline A. siamensiae & SS 81.02 & - & EF175666 & EF175645 \\
\hline Asterina cestricola & TH 591 & - & GU586215 & GU586209 \\
\hline A. fuchsiae & TH 590 & - & GU586216 & GU586210 \\
\hline A. phenacis & TH 589 & - & GU586217 & GU586211 \\
\hline A. weinmanniae & TH 592 & - & GU586218 & GU586212 \\
\hline A. zanthoxyli & TH 561 & - & GU586219 & GU586213 \\
\hline Asterotexis cucurbitacearum & VIC 24814 & - & KР143734 & - \\
\hline A. cucurbitacearum & PMA M 0141224 & - & HQ610510 & - \\
\hline Cenococcum geophilum & CG54 & KC967410 & JN860134 & JN860120 \\
\hline Delitschia chaetomioides & SMH 3253.2 & - & GU390656 & - \\
\hline D. winteri & CBS 225.62 & - & DQ678077 & DQ678026 \\
\hline Gloniopsis praelonga & CBS 112415 & EU552133 & FJ161173 & FJ161134 \\
\hline Glonium stellatum & CBS 207.34 & - & FJ161179 & FJ161140 \\
\hline Hysterium angustatum & CBS 236.34 & KX611363 & FJ161180 & GU397359 \\
\hline Hysterobrevium smilacis & CBS 114601 & - & FJ161174 & FJ161135 \\
\hline Jahnula aquatica & R $68-1$ & JN942354 & EF175655 & EF175633 \\
\hline Manglicola guatemalensis & BCC 20156 & JN819283 & FJ743448 & FJ743442 \\
\hline M. guatemalensis & BCC 20079 & JN819282 & FJ743449 & FJ743443 \\
\hline Massaria anomia & CBS 591.78 & HQ599380 & GU301839 & GU296169 \\
\hline M. gigantispora & M 26 & HQ599399 & HQ599397 & HQ599447 \\
\hline M. inquinans & M 19 & MH875187 & HQ599402 & HQ599444 \\
\hline $\begin{array}{l}\text { Minutisphaera } \\
\text { parafimbriatispora }\end{array}$ & G156-4a & KР309991 & КР309996 & KP310002 \\
\hline Minutisphaera aquaticum & $\begin{array}{l}\text { MFLUCC 19- } \\
0497\end{array}$ & MN85718 & MN857176 & - \\
\hline M. parafimbriatispora & G156-4b & КР309992 & КР309997 & KP310003 \\
\hline M. aspera & G427-1a & КР309989 & MH878174 & КР309999 \\
\hline M. aspera & G427-1b & КР309990 & NG060319 & KP310000 \\
\hline M. fimbriatispora & A242-7d & JX474872 & HM196366 & HM196373 \\
\hline M. fimbriatispora & G155-1a & JX474874 & JX474859 & JX474865 \\
\hline M. japonica & JCM 18562 & AB733436 & AB733439 & AB733433 \\
\hline M. japonica & JCM 18560b & NR119419 & AB733440 & AB733434 \\
\hline
\end{tabular}


Table 1 Continued.

\begin{tabular}{lllll}
\hline \multirow{2}{*}{ Taxa } & \multirow{2}{*}{ Strain no. } & \multicolumn{3}{l}{ GenBank accession numbers } \\
\cline { 3 - 5 } & & ITS & LSU & SSU \\
\hline Minutisphaera sp. & G156-1a & JX474875 & - & - \\
Minutisphaera sp. & G156-2a & JX474876 & - & - \\
Minutisphaera sp. & G156-2b & JX474877 & - & - \\
Myrmaecium rubricosum & CBS 139067 & MG708367 & KP687881 & KP687977 \\
M. rubrum & CBS 109505 & MH862829 & GU456324 & GU456303 \\
Mytilinidion acicola & EB O349 & - & GU323209 & GU323185 \\
M. andinense & CBS 123562 & - & FJ161199 & FJ161159 \\
M. mytilinellum & CBS 303.34 & HM163570 & FJ161184 & FJ161144 \\
Oedohysterium insidens & CBS 238.34 & - & FJ161182 & FJ161142 \\
Psiloglonium araucanum & CBS 112412 & - & FJ161172 & FJ161133 \\
\hline
\end{tabular}

\section{Results}

\section{Phylogenetic analyses}

The aligned sequence matrix comprises LSU (1321 bp), SSU (1167) and ITS (486 bp) sequence data for 41 taxa from six orders (Asterinales, Hysteriales, Jahnulales, Minutissphaerales, Mytilindiales and Pleosporales) including two outgroup taxa Myrmaecium rubricosum (CBS 139067) and Myrmaecium rubrum (CBS 109505). The combined gene analysis comprising 2973 characters after alignment (including gaps), of which 267 were parsimony-informative, 675 were parsimony-uninformative and 2121 characters were constant. The RAxML analysis of the combined dataset yielded the best scoring tree (Fig. 1) with a final ML optimization likelihood value of -14936.216419 . The matrix had 975 distinct alignment patterns, with $26.56 \%$ undetermined characters or gaps. Estimated base frequencies were as follows: $\mathrm{A}=0.257773, \mathrm{C}=$ $0.221588, \mathrm{G}=0.281573, \mathrm{~T}=0.239066$; substitution rates $\mathrm{AC}=1.252928, \mathrm{AG}=3.119769, \mathrm{AT}=$ 1.187559, CG $=1.297978, \mathrm{CT}=8.312917, \mathrm{GT}=1.000000$; gamma distribution shape parameter $\alpha$ $=0.004255$. RAxML, MP and Bayesian analyses of the combined dataset resulted in phylogenetic reconstructions with largely similar topologies and the RAxML tree is shown in Fig. 1. Bootstrap support values for RAxML and MP greater than $75 \%$ and Bayesian posterior probabilities greater than 0.95 are given at each node (Fig. 1).

In the phylogenetic analyses, all the strains of Minutisphaera clustered together with strong support (100 ML/, 100/MP and 1.00 PP). The novel species Minutisphaera aquaticum clustered with members of Minutisphaera, but in a distinct lineage with good bootstrap support (88\% MLBS/ 75 \% MPBS and 1.00 BYPP, Fig. 1).

\section{Taxonomy}

Minutisphaera aquaticum D.F. Bao, Z.L. Luo, K.D. Hyde \& H.Y. Su, sp. nov.

Fig. 2 Index Fungorum number: IF557049; Facesoffungi number: FoF 07084

Etymology - Referring to the aquatic habitat from which the fungus was collected.

Holotype - MFLU 19-2846

Saprobic on decaying wood, submerged in freshwater habitats. Sexual morph: Ascomata 71$90 \times 85-98 \mu \mathrm{m}(\bar{x}=80.5 \times 91 \mu \mathrm{m}, \mathrm{n}=5)$ diam, superficial, scattered, globose, dark brown to black. Ostiole absent. Peridium 12-17 $\mu$ m wide, comprises two layers, outer layers composed of dark brown cells of textura angularis, inner layer composed rectangular to subglobose hyaline cells. Hamathecium composed of 1.2-2.0 $\mu \mathrm{m}$ wide, cellular pseudoparaphyses, sparse in young ascomata, becoming abundant with age. Asci 52-64 × 21.5-27.5 $\mu \mathrm{m}(\bar{x}=58 \times 23 \mu \mathrm{m}, \mathrm{n}=20)$, 8spored, bitunicate, fissitunicate, obovoid to broadly cylindrical, sessile to short pedicellate, without ocular chamber. Ascospores 28-32 × 7.6-8.7 $\mu \mathrm{m}(\bar{x}=30 \times 8 \mu \mathrm{m}, \mathrm{n}=30)$, bi-seriate, hyaline, 
fusiform to clavate, straight, curved at both of ends, with a supra-median primary septum, constrict at the septum, upper cell wider and shorter than lower cell, without or with 1-2 large guttules when young, multi-guttulate when mature, smooth-walled, with or without sheath and appendages. Asexual morph: Undetermined.

Material examined - THAILAND, That Phanom, Nakhon Phanom province, on submerged decaying wood in the Mekong River, 13 November 2018, D.F. Bao, B-163 (MFLU 19-2846, holotype), ex-type culture, MFLUCC 19-0497.

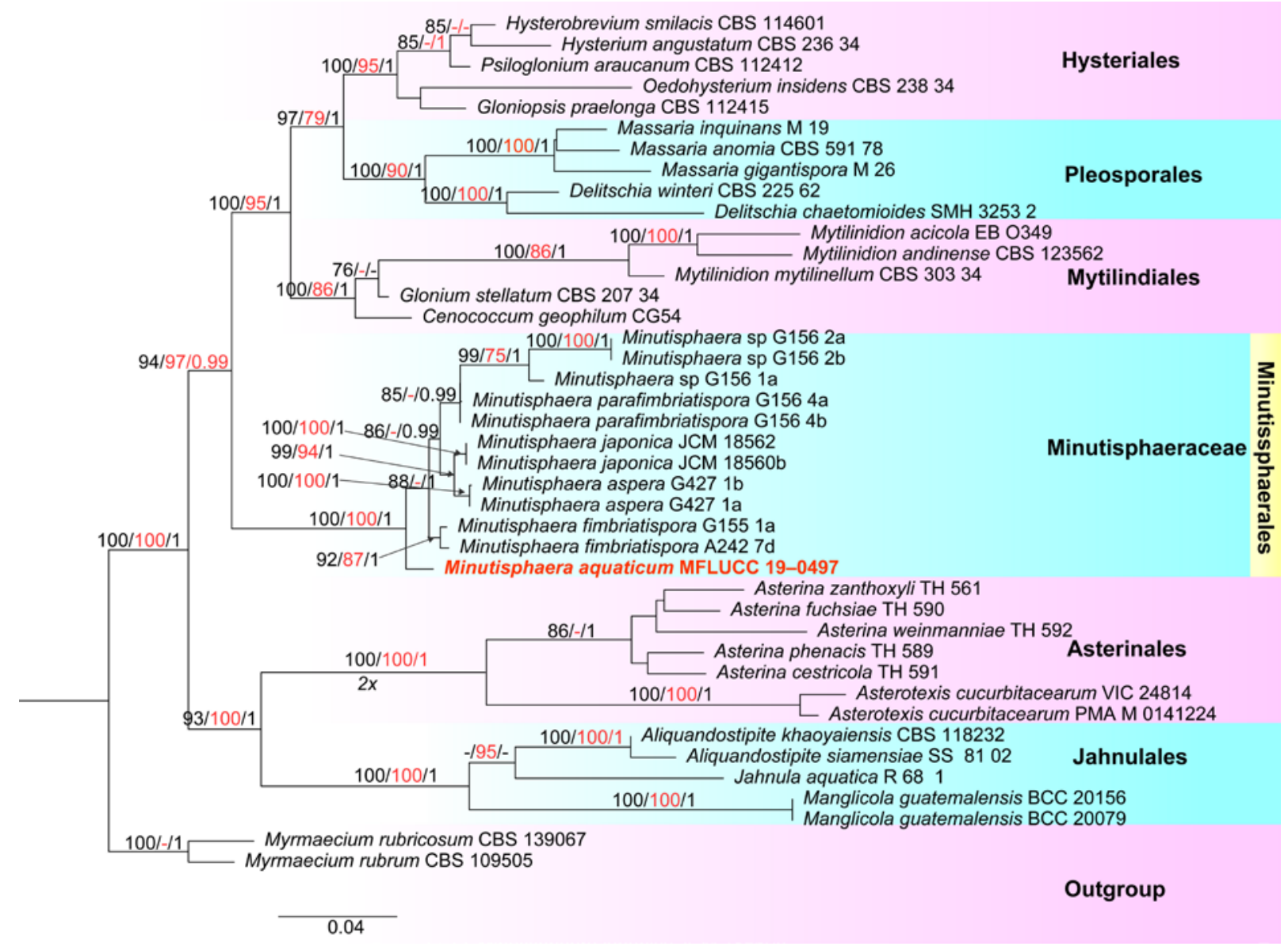

Fig. 1 - Phylogenetic tree based on RAxML analyses of a combined LSU, SSU and ITS dataset. Bootstrap support values for maximum likelihood (ML, black) and maximum parsimony (MP, blue) higher than $75 \%$ and Bayesian posterior probabilities (BYPP, red) greater than 0.95 are indicated above the nodes as MPBS / MLBS /PP. The tree is rooted with Myrmaecium rubricosum (CBS 139067) and Myrmaecium rubrum (CBS 109505). The new isolate is in bold and red.

\section{Discussion}

In this study, we introduce a new species, Minutisphaera aquaticum with morphological and phylogenetic evidences. Minutisphaera aquaticum is most similar to $M$. fimbriatispora and $M$. parafimbriatispora in having superficial, globose ascomata, obovoid to broadly cylindrical, sessile to short pedicellate asci and fusiform ascospores with a supramedian primary septum. Minutisphaera fimbriatispora and $M$. parafimbriatispora share similar morphological characters and, Raja et al. (2015) distinguished these two species based on the size of asci and ascospores. Minutisphaera aquaticum can be distinguished from these two species by the size of ascomata, asci and ascospores (Table 2). While ascospores of $M$. fimbriatispora and $M$. parafimbriatispora have both sheaths and appendages, ascospores of M. aquaticum lacks appendages. 

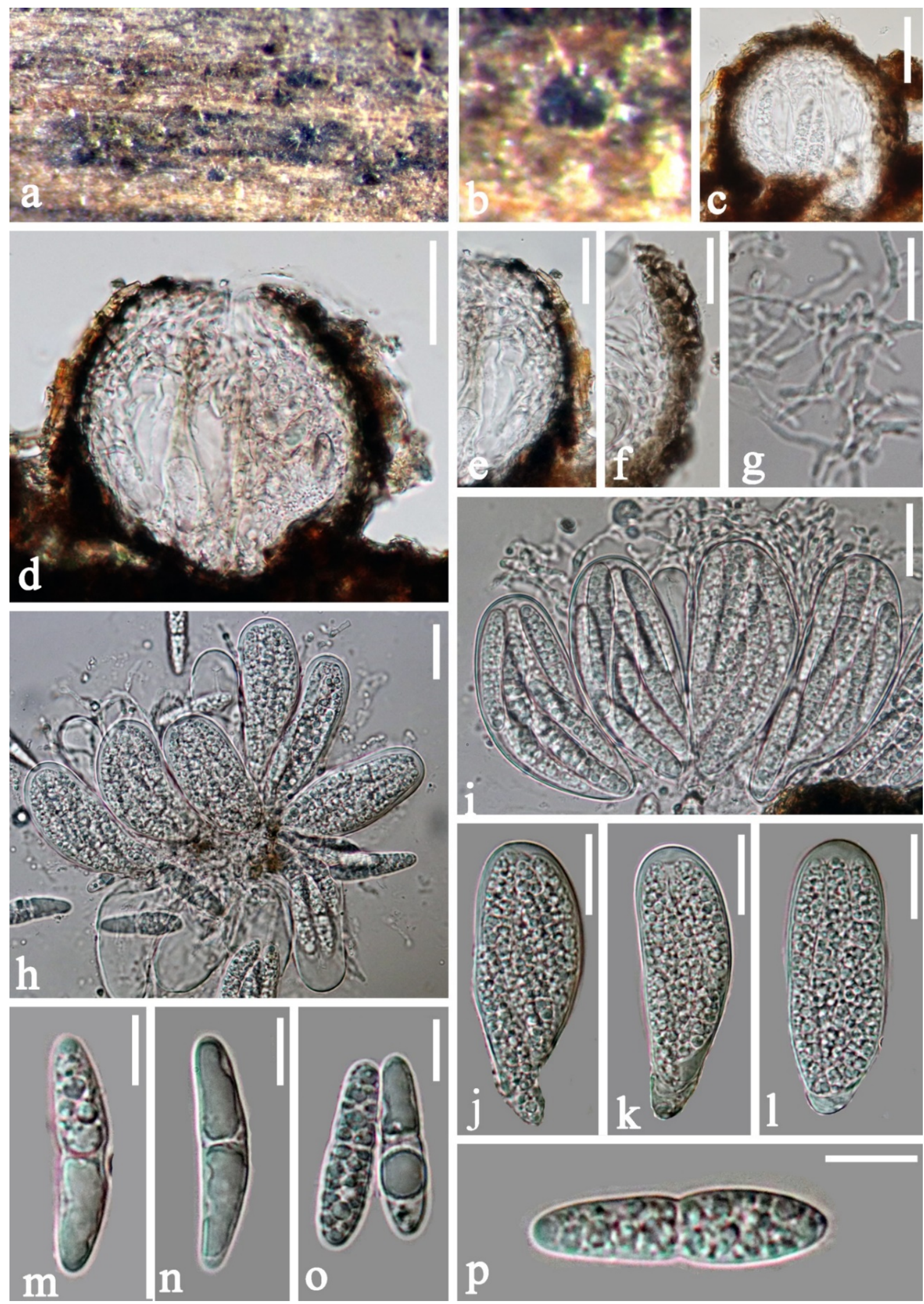

Fig. 2 - Minutisphaera aquaticum (MFLU 19-2846, holotype). a, b Ascomata on submerged wood. c, d Sections of ascoma. e, f Peridium. g Pseudoparaphyses. h-l Asci. m-p Ascospores. Scale bars: $\mathrm{c}, \mathrm{d}=30 \mu \mathrm{m}, \mathrm{e}-\mathrm{l}=20 \mu \mathrm{m}, \mathrm{m}-\mathrm{p}=10 \mu \mathrm{m}$.

Minutisphaera aquaticum can be distinguished from $M$. aspera and $M$. japonica by its ascospores in having hyaline, fusiform to clavate, smooth-walled ascospores with curved ends. 
Ascospores of M. aspera are broadly fusiform when young, becoming ellipsoidal with age, roughwalled and dark brown when mature. In addition, Minutisphaera aquaticum differs from $M$. japonica in having fusiform to clavate, straight ascospores with curved ends. However, ascospores of $M$. japonica are broadly fusiform, slightly curved, acute at the apex and rounded at the base.

In the phylogenetic study by Raja et al. (2015), Minutisphaera formed a monophyletic clade in the Dothideomycetes and did not share any relationship with the order of Dothideomycetes. They, therefore, introduced a new order Minutisphaerales to accommodate Minutisphaera species. In our phylogenetic study, Minutisphaera species formed a distinct clade, sister to Mytilinidiales with strong bootstrap support (100 \% MLBS, $95 \%$ MPBS and 1.00 BYPP). This result is similar to the previous studies and supports the placement of Minutisphaerales (Raja et al. 2015).

Lignicolous freshwater fungi have the ability to decay submerged, waterlogged woody debris, (Yuen et al. 1998, Bucher et al. 2004). Members of this family probably decompose lignocellulose in the woody litter, softening the wood, and enhanced nutrient cycling (Wong et al. 1988). Species of Minutisphaera are saprobic and have only been reported from submerged wood in freshwater habitats (Ferrer et al. 2011, Raja et al. 2013, 2015). Our study also indicates that Minutisphaera species are restricted to freshwater ecosystems and shows the genus to be diverse. Thus, further studies in different regions should also reveal novel species. Morphologically, all the species of this genus have sheaths or having both sheaths and appendages. The formation of sheath and appendages may be related to the habitat (Moss 1990, Jones 1995, Shearer 1993, 2001), where they enhanced the ascospores in attaching to substrates in moving water and aid in dispersal (Shearer 1993, Hyde \& Goh 2003, Jones 2006).

Table 2 Size of ascomata, asci and ascospores comparisons of Minutisphaera species in this study

\begin{tabular}{llll}
\hline Taxa & Ascomata $(\boldsymbol{\mu m})$ & Asci $(\boldsymbol{\mu m})$ & Ascospores $(\boldsymbol{\mu m})$ \\
\hline Minutisphaera fimbriatispora & $110-120 \times 120-$ & $52-97 \times 18-31$ & $24-36 \times 6-8$ \\
& 150 & & \\
M. parafimbriatispora & $160-170 \times 170-180$ & $48-72 \times 15-22$ & $18-25 \times 4-7$ \\
M. aquaticum & $71-90 \times 85-98$ & $52-64 \times 21.5-27.5$ & $28-32 \times 7.6-8.7$ \\
M. japonica & $90-130 \times 150-300$ & $55-82.5 \times 21.5-$ & $25-33 \times 9-11$ \\
M. aspera & & 32.5 & \\
\hline
\end{tabular}

\section{Key to species of Minutisphaera}

1. Ascospores with appendages 2

1. Ascospores lacking appendages 3

2. Ascospores18-25 × 4-7 $\mu \mathrm{m}$

2. Ascospores $24-36 \times 6-8 \mu \mathrm{m}$ Minutisphaera parafimbriatispora Minutisphaera fimbriatispora

3. Ascospores with verruculose wall and thicker septate when mature

Minutisphaera aspera

3. Ascospores with smooth wall 4

4. Ascospores, broadly fusiform, slightly curved, acute at the apex, rounded at the base

4. Ascospores fusiform to clavate, straight, smooth walled, with curved ends

Minutisphaera japonica

Minutisphaera aquaticum

\section{Acknowledgements}

We would like to thank the National Natural Science Foundation of China (Project ID: 31660008 and 31860006), Fungal diversity conservation and utilization innovation team of Dali University (ZKLX2019213) and the Thailand Research Fund grant "impact of climate change on fungal diversity and biogeography in the Greater Mekong Sub-region (RDG6130001)” for financial 
and laboratory support. Dan-Feng Bao thanks Shaun Pennycook from Landcare Research, Auckland, New Zealand, for advising on the taxon names, Wen-Li Li, Yan-Mei Zhang and HongWei Shen are acknowledged for their help on DNA extraction and PCR amplification.

\section{References}

Bucher VVC, Hyde KD, Pointing SB, Reddy CA. 2004 - Production of wood decay enzymes, mass loss and lignin solubilization in wood by diverse freshwater fungi. Microb Ecol 48, 331-337.

Chomnunti P, Hongsanan S, Aguirre-Hudson B, Tian Q et al. 2014 - The sooty moulds. Fungal Diversity 66, 1-36.

Ferrer A, Miller AN, Shearer CA 2011 - Minutisphaera and Natipusilla: two new genera of freshwater Dothideomycetes. Mycologia 103, 411-423.

Hillis DM, Bull JJ. 1993 - An empirical test of bootstrapping as a method for assessing confidence in phylogenetic analysis. Systematic Biology 42, 182.

Huelsenbeck JP, Ronquist F. 2001 - MRBAYES: Bayesian inference of phylogenetic trees. Bioinformatics 17, 754-755.

Hyde KD, Fryar S, Tian Q, Bahkali AH et al. 2016 - Lignicolous freshwater fungi along a northsouth latitudinal gradient in the Asian/Australian region; can we predict the impact of global warming on biodiversity and function? Fungal Ecology 19, 190-200.

Hyde KD, Goh TK. 2003 - Adaptations for dispersal in filamentous freshwater fungi. Fungal Diversity 10, 231-258.

Jayasiri SC, Hyde KD, Ariyawansa HA, Bhat DJ et al. 2015 - The faces of fungi database: fungal names linked with morphology, phylogeny and human impacts. Fungal Diversity 74, 3-18.

Jones EBG. 1995 - Ultrastructure and taxonomy of the aquatic ascomycetous order Halosphaeriales. Can J Bot 73, 790-801.

Jones EBG. 2006 - Form and function of fungal spore appendages. Mycoscience 47, 167-183.

Katoh K, Standley DM. 2013 - MAFFT multiple sequence alignment software version 7: improvements in performance and usability. Molecular biology and evolution 30, 772-780.

Larget B, Simon DL. 1999 - Markov Chain Monte Carlo algorithms for the Bayesian analysis of phylogenetic trees. Molecular Biology and Evolution 16, 750-759.

Liu JK, Hyde KD, Jeewon R, Phillips AJL et al. 2017 - Ranking higher taxa using divergence times: a case study in Dothideomycetes. Fungal Diversity 84, 75-99.

Luo ZL, Hyde, KD, Liu JK, Bhat DJ et al. 2018 - Lignicolous freshwater fungi from China II: Novel Distoseptispora (Distoseptisporaceae) species from northwestern Yunnan Province and a suggested unified method for studying lignicolous freshwater fungi. Mycosphere 9, 444-461.

Miller MA, Pfeiffer W, Schwartz T. 2010 - Creating the CIPRES Science Gateway for inference of large phylogenetic trees. In: Proceedings of the 2010 Gateway Computing Environments Workshop (GCE), New Orleans, Louisiana, November 2010, 1-8.

Moss ST. 1990 - The relevance of scanning electron microscopy to the taxonomy of marine ascomycetes. In: Glaugher D (ed) Scanning electron microscopy in taxonomy and functional morphology. Systematics Association Special 41, 149-170.

Nylander JAA. 2004 - MrModeltest v2 Program distributed by the author. Evolutionary Biology Centre, Uppsala University, Uppsala.

Raja HA, El-Elimat T, Oberlies NH, Shearer CA et al. 2015 - Minutisphaerales (Dothideomycetes, Ascomycota): a new order of freshwater ascomycetes including a new family, Minutisphaeraceae, and two new species from North Carolina, USA. Mycologia 107, 845862.

Raja HA, Oberlies NH, Figueroa M, Tanaka K et al. 2013 - Freshwater ascomycetes: Minutisphaera (Dothideomycetes) revisited, including one new species from Japan. Mycologia 105, 959-976. 
Rannala B, Yang Z. 1996 - Probability distribution of molecular evolutionary trees: a new method of phylogenetic inference. Journal of Molecular Evolution 43, 304-311.

Shearer CA. 1993 - The freshwater Ascomycetes. Nova Hedwigia 56, 1-33.

Shearer CA. 2001 - The distribution of freshwater filamentous ascomycetes. In: Misra JK, Horn BW (eds) Trichomycetes and other fungal groups. Science Publishing, Enfield, NH, pp 225292.

Stamatakis A, Hoover P, Rougemont J. 2008 - A rapid bootstrap algorithm for the RAxML webservers. Systematic Biology 75, 758-771.

Stamatakis A. 2006 - RAxML-VI-HPC: maximum likelihood-based phylogenetic analyses with thousands of taxa and mixed models. Bioinformatics 22, 2688-2690.

Swofford DL. 2003 - PAUP*: Phylogenetic analysis using parsimony (*and other methods). Version 4. Sinauer, Sunderland.

Vilgalys R, Hester M. 1990 - Rapid genetic identification and mapping of enzymatically amplified ribosomal DNA from several Cryptococcus species. Journal of Bacteriology 172, 4238-4246.

White TJ, Bruns T, Lee S, Taylor J. 1990 - Amplification and direct sequencing of fungal ribosomal RNA genes for phylogenetics. In: Innis GM, Shinsky D, White T (eds) PCR protocols: a guide to methods and applications. Academic, New York, 315-322.

Wong KMK, Goh TK, Hodgkiss IJ, Hyde KD et al. 1998 - Role of fungi in freshwater ecosystems. Biodiversity, Conservation 7, 1187-1206.

Yuen TK, Hyde KD, Hodgkiss IJ. 1998 - Physiological growth parameters and enzyme production in tropical freshwater fungi. Mater Org 32, 2-16.

Zhaxybayeva O, Gogarten JP. 2002 - Bootstrap, Bayesian probability and maximum likelihood mapping: exploring new tools for comparative genome analyses. BMC Genomics 3, 4. 